\title{
Use of Ørsted scalar data in evaluating the pre-Ørsted main field candidate models for the IGRF 2000
}

\author{
Mioara Mandea and Benoit Langlais \\ Institut de Physique du Globe, B.P. 89, 4 Place Jussieu, 75252 Paris cedex 5, France
}

(Received February 14, 2000; Revised June 26, 2000; Accepted June 26, 2000)

\begin{abstract}
For describing the main field model at the 2000.0 epoch and the secular variation over the 2000-2005 timespan, three candidate models for the International Geomagnetic Reference Field (IGRF 2000) were proposed at the beginning of 1999, called in alphabetical order IPGP00 (proposed by IPGP), IZMI00 (proposed by IZMIRAN) and USUK00 (proposed by USGS/BGS). A fourth model, IGRF95 (the updated IGRF 1995), was suggested by the Working Group chairman. The modelling methods and the data used are presented by each team elsewhere in this special issue. This study is an attempt to test these models using the total field intensity provided by the Ørsted satellite, the only data available from that satellite at the time when the two tests describing here were done. The first test consists of evaluating the differences between the real and the synthetic data computed from the candidate models. The second test compares the capability of the candidate models to reduce the Backus effect, using a predictive dip-equator position and Ørsted data. Both tests show that the quality of the candidate models is far from being acceptable, and, therefore, a new candidate model for the main field, using vectorial Ørsted data, is required.
\end{abstract}

\section{Introduction}

A five-year interval separates the epochs for which models to describe the main geomagnetic field and its secular variation are proposed by Working Group V-8 of the International Association of Geomagnetism and Aeronomy (IAGA). To produce these models, the standard procedure consists of a request for candidate models from the geomagnetic community, followed by an evaluation of the models submitted, and finally an adoption of the final model, the so-called International Geomagnetic Reference Field (IGRF). In this paper the quality of the models submitted prior to the availability of Ørsted vectorial data is examined.

The most relevant information about the candidate models are summarized here. The full description of the inversion method, and the geomagnetic data used, are described by each team in the present issue. The four models, up to degree and order 10, are referred to as IPGP00, IZMI00, USUK00, and IGRF95.

The IPGP00 model was proposed by B. Langlais and M. Mandea (Institut de Physique du Globe de Paris, France). The model was based on 1997 observatory annual means (145 values of $X, Y, Z$ components), repeat stations occupied during 1997 and adjusted to 1997.5 using the nearby observatory (67 values of $X, Y, Z$ components), and marine data obtained from 1995 to 1998, at local night time and for $k_{p} \leq 2$ and then reduced to 1997.5 by the IPGP00 secular variation model (139 values of $F$ component). The repeat stations and the marine data were weighted 2/3 compared to observatories. All data sites were given weights depending on their geographical distribution. The main field model

Copy right(C) The Society of Geomagnetism and Earth, Planetary and Space Sciences (SGEPSS); The Seismological Society of Japan; The Volcanological Society of Japan; The Geodetic Society of Japan; The Japanese Society for Planetary Sciences. was produced by a least square technique for 1997.5 epoch and then extrapolated to 2000.0 using the IPGP00 secular variation model.

The IZMI00 model was proposed by V. P. Golovkov et al. (IZMIRAN, Russia). The main field model and the secular variation one were obtained simultaneously. Only data from geomagnetic observatories were used, available for 1996 onwards (217 annual means for $X, Y, Z$ components). From 1970 to 1995,162 values for the $X, Y, Z$ components were synthesized for every second year from the IZMST 70-95 model, on a grid of $20^{\circ} \times 20^{\circ}$. Unit weights were applied to all data. Time functions were obtained from 28 well-distributed observatories, which were then imposed in a spherical harmonic analysis applied to all the data.

The USUK00 model was proposed by J. M. Quinn (US Navy, USA) and S. Macmillan (British Geological Survey, UK). The model was built using three datasets from repeat stations, aeromagnetic surveys and synthetic data. The 2675 $X, Y, Z, F$ repeat station measurements over 1988-1998 were adjusted to 1995.0 using the previous USGS/BGS secular variation model up to 1990, and the present BGS model beyond 1990. The aeromagnetic dataset consisted of 41000 $F$-observations, from 1988 to 1998 , updated as for the repeat station data. The synthetic values were computed on a $2^{\circ} \times 2^{\circ}$ grid from the 1992.5 model of Quinn et al. (1995), and updated to 1995.0 by the BGS secular variation model. The relative data quality was assessed by giving different weights to each dataset: 0.2 to repeat station data, 0.06 to aeromagnetic data and 1 to synthetic data. The model produced for epoch 1995.0 was extrapolated to 2000.0 using the present BGS secular variation model.

The fourth model, IGRF95, was simply the IGRF 1995 model updated using the IGRF 1995 secular variation model.

The tests presented in this paper were done before the 
XXII IUGG General Assembly (July 1999) in preparation for the Working Group V-8 meeting of IAGA, when discussions about the adoption of the IGRF 2000 model were held (hence the reason that the selected Ørsted data for testing the candidate models covers a time interval prior to June 1999).

\section{Principle of Testing}

Different procedures have been applied in the past, and the reader is referred to the special issues of Physics of the Earth and Planetary Interiors $(\mathbf{4 8}, 1987)$ or Journal of Geomagnetism and Geoelectricity (34, 1982; 44, 1992; 49, 1997). The advantages and disadvantages of the procedures applied to verify the quality of the models were pointed out by Langel (1992). Two main approaches are possible: to compare the models among themselves and to compare the models with real data.

In our evaluation of the four candidate models, we test the predictive power of each model versus real data. To be sure that the dataset used for comparison is independent from all data used in computing the candidate models, the Ørsted scalar data were chosen. These data present two important advantages: there is no link with the data used by the three teams in deriving their models, and Ørsted data are welldistributed at the Earth's surface, so the models could be tested over global and regional areas.

Two tests were carried out. First, we evaluated the differences between the Ørsted data and synthetic data computed from the candidate models. Second, we tested the candidate models via their capability to predict a dip equator in order to reduce the Backus effect when inverting together with Ørsted data, by applying the method proposed by Khokhlov et al. (1997, 1999).

\section{Remark}

Tests were performed by reducing Ørsted data to the epoch of the models, using the candidate secular variation models up to degree and order 8. Tests using the IGRF 1995 secular variation model to reduce Ørsted data were also performed, but with worse results, not shown in this paper.

\section{3. Ørsted Data Selection}

The Danish Ørsted satellite was launched on February 23rd 1999, by NASA, and data became available on March 15th. After numerous problems, mostly linked to time shifts between instruments, the scalar data were made accessible via the web site of the Danish Meteorological Institut (DMI, $h t t p: / / w w w . d m i . d k)$. The data were presented in a raw format. Some problems arose because the pre-launch calibration was applied to the data, and some errors in the timing of the data still existed. We re-corrected the data, using the in-flight calibration, kindly furnished by the Ørsted Science Data Center (OSDC). This correction corresponded to a shift of about $1.5 \mathrm{nT}$. Tests were also performed to assess the validity and the consistency of the data (Langlais et al., 1999; Appendix of Lowes et al., 2000).

After the data were corrected for in-flight calibration and checked, the time-span from March 20th to April 26th was considered. Over this time interval data were selected in the usual way, using local time and magnetic activity indices. The first choice was to select only night-side data, in order to reduce the diurnal variation. Thereafter the three hour time

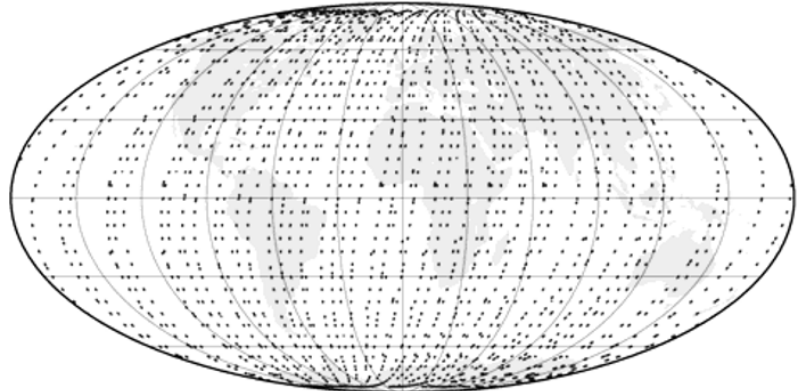

Fig. 1. Distribution of Ørsted data (19457 field intensity values), known as set1 (Mollweide equal area projection).

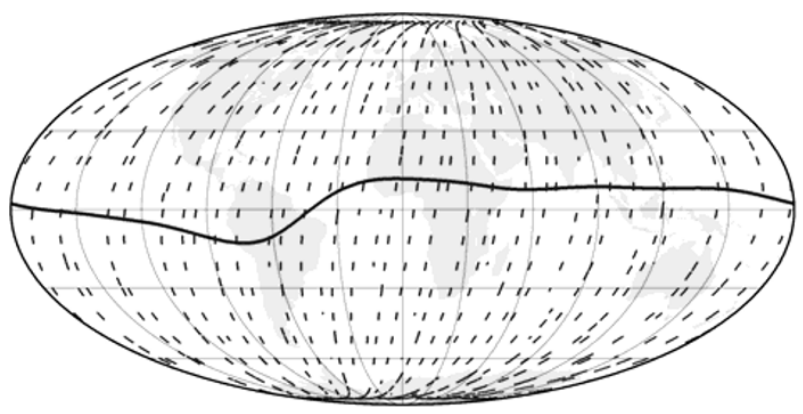

Fig. 2. Distribution of Ørsted data (6149 field intensity values), known as set 2 , together with position of the dip equator predicted by USUK 00 .

intervals over which the $K_{p}=0_{0}$ or $K_{p}=0_{+}$were selected.

Considering these selection criteria, 232637 scalar measurements were retained. This set was reduced to two smaller ones, in order to have homogeneous data distributions. The first one (setl) consisted of a maximum of 10 measurement points per $5^{\circ} \times 5^{\circ}$, containing 19457 field intensity values. The second one (set 2 ) consisted of a maximum of 10 measurement points per $10^{\circ} \times 10^{\circ}$, and contained 6149 field intensity values. Each of these datasets was used for a different test. As the candidate models were built for epoch 2000.0, the Ørsted data were updated to this epoch, using the secular variation of each candidate model, respectively. The data distributions of these two datasets are shown in Figs. 1 and 2 .

\section{Results of Tests}

4.1 How well do the models predict the field intensity?

The aim of this first test is to show the capability of the submitted models to predict the intensity of the Earth's magnetic field at the satellite elevation.

Synthetic data for the 19457 measurement locations of set 1 were computed from the candidate models (all up to degree and order 10) and compared with the real Ørsted data. The results, in terms of mean and standard deviation between real and synthetic data, are presented in Table 1.

For each model the resulting pattern of residuals is shown approximately by the appropriate contour plot of Fig. 3 . 

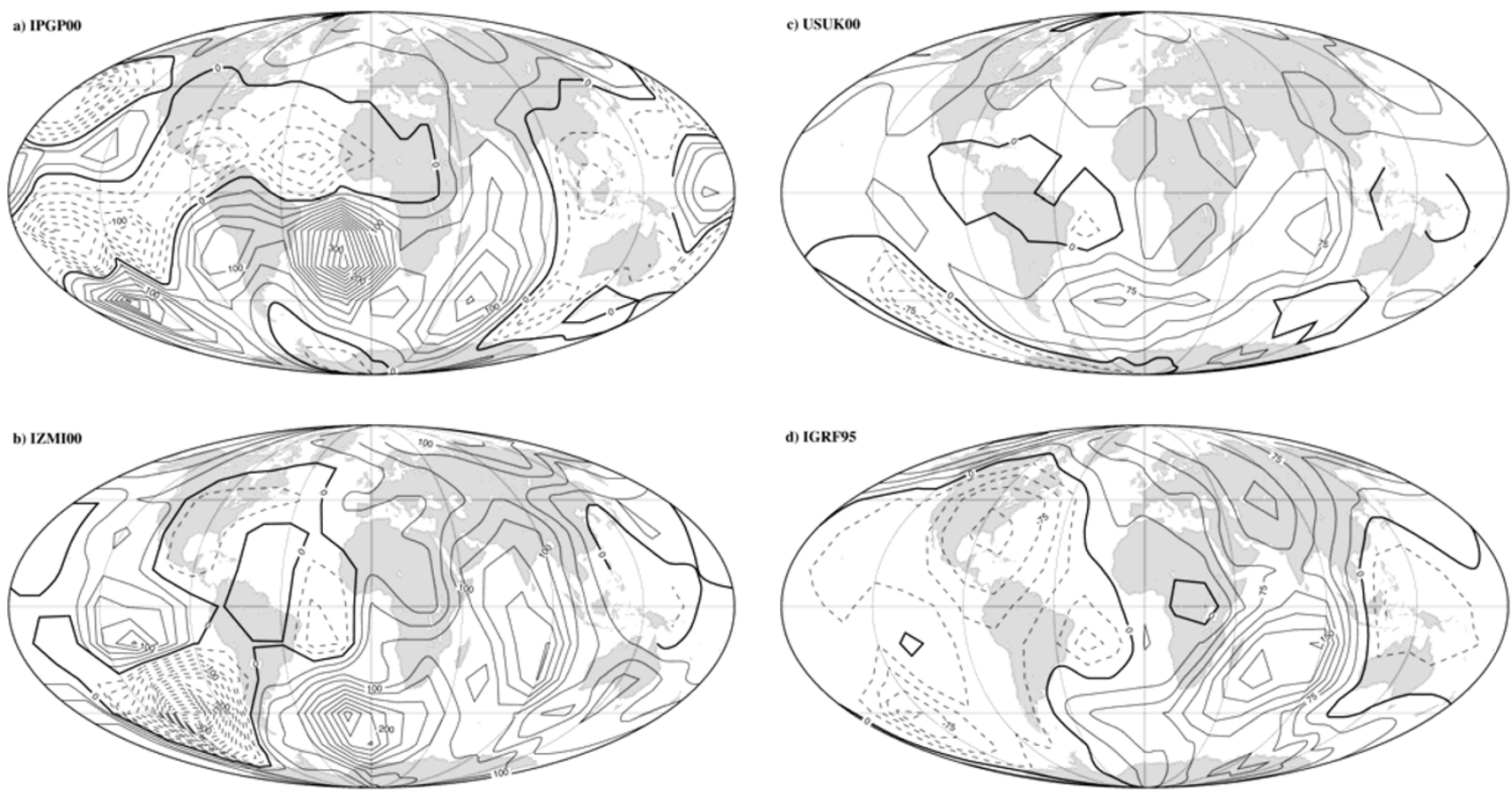

Fig. 3. Residuals between Ørsted scalar data and predicted one from a) IPGP00, b) IZMI00, c) USUK00, d) IGRF95. Residuals are averaged on a grid of $15^{\circ} \times 15^{\circ}$, resulting 288 points then contoured, with a contour spacing of $25 \mathrm{nT}$.

Table 1. Comparison between real and synthetic data for set1.

\begin{tabular}{ccc}
\hline Model & Mean $(\mathrm{nT})$ & $\sigma(\mathrm{nT})$ \\
\hline IPGP00 & 22.4 & 65.6 \\
IZMI00 & 46.6 & 82.1 \\
USUK00 & 23.7 & 30.5 \\
IGRF95 & 27.2 & 61.5 \\
\hline
\end{tabular}

\subsection{How well do the models predict the dip equator?}

Let us briefly recall some aspects concerning the derivation of a main field model using only scalar data. Such a model cannot be obtained properly, and it is strongly contaminated by the Backus effect: this is due to the non-uniqueness of the solution because the orientation of the field is not known (Backus, 1970). This problem can be solved using the method proposed by Khokhlov et al. $(1997,1999)$ with a numerical application presented in Ultré-Guérard et al. (1998). These studies show that, given the position of the dip equator with a reasonable accuracy, it is possible to recover a main field model without any Backus effect.

We were then interested by the coherence of the dip equator predicted by the four models with the Ørsted field intensity. Dip equators were simulated for the mean epoch of the available Ørsted data, i.e. March 28th 1999, using main field candidate models (up to degree and order 10) and their secular variation models (up to degree and order 8 ). Then an inversion was performed, using the Ørsted dataset set 2 and the 720 locations where $B_{r}$ is null (one point every $0.5^{\circ}$ of longitude) on a sphere of radius $6371.2 \mathrm{~km}$. The inversion was performed using the least square method of Cain et al. (1967), with unit weights for equatorial data and weights based on sine of colatitude for scalar satellite data, described as follows:

$$
\begin{gathered}
\omega_{i}^{\text {sca }}=\frac{2}{3} \frac{N_{\text {equ }}+N_{\text {sca }}}{T_{\text {sca }}} \sin \left(\theta_{i}\right) \\
\omega_{j}^{\text {equ }}=\frac{1}{3} \frac{N_{\text {equ }}+N_{\text {sca }}}{T_{\text {equ }}}
\end{gathered}
$$

with

$$
\begin{gathered}
T_{\text {sca }}=\sum_{i=1}^{N_{\text {sca }}} \sin \left(\theta_{i}\right) \\
T_{\text {equ }}=N_{\text {equ }}
\end{gathered}
$$

where $\omega_{i}^{\text {sca }}$ denotes the weight of the $i$ th scalar data of colatitude $\theta_{i}$ and $\omega_{j}^{\text {equ }}$ denotes the weight of the $j$ th equatorial data. $N_{\text {sca }}$ and $N_{\text {equ }}$ are the total number of scalar and equatorial data, i.e. 6149 and 720 .

The distribution of the scalar dataset used, together with the position of the dip equator predicted by USUK00, is shown in Fig. 2. The residuals given by these inversions up to degree and order 10 are summarised in Table 2. For each combined candidate equator/Ørsted model (inversion) rms are given separately for the $\Delta F$ between that model and the $\emptyset$ rsted data $\left(\mathrm{RMS}_{\mathrm{sca}}\right)$, and for the vertical field of that Ørsted model at the equator locations given by the candidate models, where the vertical field would be zero $\left(\mathrm{RMS}_{\text {equ }}\right)$. The values of rms for the combined datasets (weighted according to Eqs. (1) and (2)) are also given ( $\mathrm{RMS}_{\text {tot }}$ ).

Results of this test have to be careful considered: a wrong model could be considered closer to the reality if only the scalar residuals or only the equatorial residuals are taken into account. Let the main field model $M_{0}$ be the true, complicated field model, but slightly noised, and two other models 
Table 2. Test of the prediction of the dip equator.

\begin{tabular}{lccc}
\hline Model & $\mathrm{RMS}_{\text {sca }}(\mathrm{nT})$ & $\mathrm{RMS}_{\text {equ }}(\mathrm{nT})$ & $\mathrm{RMS}_{\text {tot }}(\mathrm{nT})$ \\
\hline IPGP00 & 10.9 & 6.0 & 9.6 \\
IZMI00 & 9.3 & 2.7 & 7.7 \\
USUK00 & 6.7 & 1.3 & 5.6 \\
IGRF95 & 7.2 & 1.5 & 6.0 \\
\hline
\end{tabular}

$M_{1}$ (the true model scaled overall by some factor significantly different from 1 ), and $M_{2}$ (a model giving the same field intensity as the true model, but corrupted by the Backus effect). Model $M_{1}$ gives the best geometry for the equator, and model $M_{2}$ better fit the scalar data, compared to model $M_{0}$, even thought $M_{0}$ is the more realistic model. These considerations show that both scalar and equatorial tests have to be performed to assess the validity of a given candidate model.

\section{Discussion and Conclusion}

Considering the tests presented in this paper, the USUK00 model best predicts the both the Ørsted intensity data and the Ørsted model dip equator. This could be related to the fact that the USUK00 model was built using a significant amount of synthetic data. The residuals given by IGRF95 are about twice as large and show a hemispherical bias (Fig. 3(d)), possibly due to error in the secular variation used to extrapolate the model from 1995; its dip equator is not quite as good. The residuals for IPGP00 and IZMI00 are comparable with one-another; the IPGP00 model gives better results when comparison is done directly versus the Ørsted scalar data, but IZMI00 better predicts the dip equator.

The residuals show that the four models could be classified into two groups: models using only real data (IPGP00 and IZMI00, for the main field models) and model using real and synthetic data (USUK00 and IGRF95). For the first class of models, the residuals vary from $-215 \mathrm{nT}$ to $+420 \mathrm{nT}$ for the IPGP00 model, and from $-475 \mathrm{nT}$ to $+320 \mathrm{nT}$ for IZMI00. For the second class, the residuals are lower, from $-105 \mathrm{nT}$ to $+110 \mathrm{nT}$ for USUK00 model, and from $-115 \mathrm{nT}$ to +205 nT for IGRF95.

The residuals obtained in testing the four candidate models for the main field clearly show that their quality is far from being acceptable. The best model, USUK00, is based on a large number of synthetic data. The two other new models used only real data, but with a poor distribution. As shown by Alexandrescu et al. (1994) and Langel et al. (1995) the data distribution is a very important parameter in main field modelling.

It is clear that new efforts must be made to improve the knowledge of the main field, so as to give an acceptable IGRF. The fact that the Ørsted satellite was flying at the time of this Working Group meeting, gave the geomagnetic community the hope to get a new and better model to be adopted as IGRF 2000. This hope was achieved in November 1999 (Lowes, 2000; Olsen et al., 2000).

Acknowledgments. We are grateful to Charlie Barton for improving the presentation of this paper, to Andy Jackson for comments on the second test and to the associate editor Frank Lowes for useful discussions. All maps were plotted using the GMT software produced by Wessel and Smith (1991). This is the IPGP contribution 1705 .

\section{References}

Alexandrescu, M., C. Ha Duyen, and J.-L. Le Mouël, Geographical distribution of magnetic observatories and field modelling, J. Geomag. Geoelectr. 46, 891-901, 1994

Backus, G. E., Non-uniqueness of the external geomagnetic field determined by surface intensity measurements, J. Geophys. Res., Space Phy., $\mathbf{7 5}$, 6339-6341, 1970

Cain, J. C., S. J. Hendricks, R. A. Langel, and W. V. Hudson, A proposed model for the International Geomagnetic Reference Field-1965, J. Geomag. Geoelectr., 19, 335-355, 1967.

Khokhlov, A., G. Hulot, and J.-L. Le Mouël, On the Backus effect-I, Geophys. J. Int., 130, 701-703, 1997.

Khokhlov, A., G. Hulot, and J.-L. Le Mouël, On the Backus effect-II, Geophys. J. Int., 137, 816-820, 1999.

Langlais, B., P. Ultré-Guérard, C. Vernin, M. Mandea, Y. Cohen, and G. Hulot, Ørsted: IPGP commissioning of the OVH magnetometer, CNES report, OERS-RP-0000-0031-IPG, 28 pp., 1999.

Langel, R. A., International Geomagnetic Reference Field: the sixth generation, J. Geomag. Geoelectr., 44, 679-707, 1992.

Langel, R. A., R. T. Baldwin, and A. W. Green, Toward an improved distribution of magnetic observatories for modelling of the main geomagnetic field and its temporal change, J. Geomag. Geoelectr. 47, 475-508, 1995.

Lowes, F. J., The working of the IGRF 2000 Task Force, Earth Planets Space, 52, this issue, 1171-1174, 2000.

Lowes, F. J., T. Bondar, V. P. Golovkov, B. Langlais, S. Macmillan, and M Mandea, Evaluation of the candidate Main Field model for IGRF 2000 derived from preliminary Ørsted data, Earth Planets Space, 52, this issue, 1183-1186, 2000

Olsen, N., T. J. Sabaka, and L. Tøffner-Clausen, Determination of the IGRF 2000 model, Earth Planets Space, 52, this issue, 1175-1182, 2000.

Quinn, J. M., R. J. Coleman, D. L. Shiel, and J. M. Nigro, The Joint US/UK 1995 epoch world magnetic model, Naval Oceanographic Office Technical Report, TR 314, 377 pp., 1995.

Ultré-Guérard, P., M. Hamoudi, and G. Hulot, Reducing the Backus effect given some knowledge of the dip-equator, Geophys. Res. Let., 16, 32013204, 1998

Wessel, P. and W. H. F. Smith, Free software helps map and display data, EOS Trans. Am. Geophys. Union, 72, 441-448, 1991.

M. Mandea (e-mail: mioara@ipgp.jussieu.fr) and B. Langlais (e-mail: langlais@ipgp.jussieu.fr) 